\title{
Squatting revisited: comparison of haemodynamic responses in normal individuals and heart transplantation recipients
}

\author{
Peter Hanson, Peter R Slane, Patricia A Rueckert, Sharon V Clark
}

\begin{abstract}
Background-Squatting produces a prompt increase in cardiac output and arterial blood pressure which is accompanied by an immediate decrease in heart rate and forearm vascular resistance. The rise in cardiac output and blood pressure has been attributed to augmented venous return from compression of leg veins, while the decreases in heart rate and forearm vascular resistance are probably due to activation of cardiopulmonary and arterial baroreflexes. Haemodynamic patterns in nine normal men and six heart transplant recipients during $2 \mathrm{~min}$ of squatting were examined to determine the role of cardiac innervation in the mediation of these responses.
\end{abstract}

Methods-Stroke volume was monitored by ensemble averaged thoracic impedance cardiography and blood pressure was determined with an Ohmeda fingertip plethysmograph. These techniques provided continuous measurements which were capable of detecting transient and non-steady state changes. Forearm blood flow was measured with venous occlusion plethysmography. Measurements were obtained after $3 \mathrm{~min}$ of quiet standing, immediately after squatting, and at 20, 60, and $120 \mathrm{~s}$ of sustained squatting.

Results-Both groups exhibited similar increases in stroke volume index (normal individuals $10.5 \mathrm{ml} / \mathrm{m}^{2}$; heart transplant recipients $10.3 \mathrm{ml} / \mathrm{m}^{2}$ ) and mean arterial pressure (normal individuals $8.5 \mathrm{~mm}$ Hg; heart transplant recipients $5.0 \mathrm{~mm}$ Hg) which were sustained throughout squatting. Each group also showed an initial decrease in peripheral resistance (normal individuals 3.6 units; heart transplant recipients $7 \cdot 7$ units) followed by a return to baseline values after $20 \mathrm{~s}$. Heart rate decreased in normal individuals ( $10 \mathrm{beats} / \mathrm{min})$ but was unchanged or minimally increased ( 2 beats $/ \mathrm{min}$ ) in heart transplant recipients. Forearm vascular resistance was conspicuously decreased in normal individuals $(47 \cdot 8$ units) but only minimally (20.9 units) and not significantly in heart transplant recipients.

Conclusions-The major haemodynamic responses to squatting (increased cardiac output and blood pressure) are similar in normal individuals and heart transplant recipients. These responses are primarily due to augmented venous return and are not altered by cardiac denervation. Both groups also exhibited a transient decline in peripheral vascular resistance which is most likely mediated by arterial baroreflexes activated by the acute rise in arterial blood pressure. The absence of a significant decrease in forearm vascular resistance in heart transplant recipients suggests that this response is partially mediated by cardiopulmonary or ventricular baroreflexes or that local forearm flow mediated vasodilatation remains impaired after heart transplantation.

(Br Heart f 1995;74:154-158)

Keywords: squatting; cardiac output; baroreflexes; transplantation

In normal individuals squatting produces a prompt rise in arterial pressure and cardiac output followed closely by moderate slowing of heart rate and an increase in forearm blood flow. ${ }^{1-4}$ The increases in cardiac output and blood pressure are attributed to the combined effects of enhanced venous return and increased vascular resistance due to compression of leg veins and arteries. ${ }^{12}$ The decline in heart rate and the forearm vasodilatation are primarily mediated by activation of inhibitory sinoaortic baroreflexes in response to the rise in blood pressure. ${ }^{12}$ In addition, cardiopulmonary receptors activated by increased central venous pressure may contribute to forearm vasodilatation. ${ }^{1}$

Orthotopic heart transplantation effectively ablates afferent and efferent innervation to the implanted sinus node, atrial cuff, and ventricles. Sinoaortic baroreflexes controlling peripheral resistance remain intact. Thus, the initial haemodynamic responses to squatting due to increased venous return should be largely preserved in heart transplant recipients. ${ }^{5}$ However, baroreflex modulation of heart rate should be absent and the contribution of cardiopulmonary baroreflexes to the control of vascular resistance may be impaired due to denervation of the allograft. ${ }^{56}$

In this study we compared the haemodynamic patterns and reflex responses of normal individuals and heart transplant recipients during squatting to determine the role of cardiac innervation in this postural manoeuvre. We used non-invasive methods which permit continuous determination of cardiac output 
and blood pressure so that transient nonsteady state differences in response could be analysed.

\section{Patients and methods \\ PATIENTS}

Nine healthy male volunteers (mean (SE) age 39 (11) years) and six orthotopic heart transplant recipients (mean (SE) age 49 (11)) were studied. Heart transplant recipients were a mean (SE) (range) of 16 (18) (3-48) months from transplantation. Their medications included routine immunosuppressive drugs (cyclosporin, prednisone, and azathioprine). Five of the heart transplant recipients were treated with antihypertensive agents (clonidine (three), enalapril (one), and nifedipine (one)). All medications were maintained during this study.

\section{MEASUREMENTS}

Cardiac output indices were measured using the CIC-1000, a computerised impedance cardiograph (version 4.2) (SORBA Medical Systems, Brookfield, Wisconsin). Impedance cardiography has been shown to provide reproducible estimates of absolute ${ }^{78}$ as well as relative $^{9}$ values for cardiac output in humans. Stroke volume and cardiac output indicates measured in transplant patients in our laboratory using the CIC-1000 are comparable to reported transplant values determined by radionuclide angiography. ${ }^{10}$

The CIC-1000 employs $R$ wave triggering and ensemble averaging which improves the signal to noise ratio by reducing respiratory and motion artefacts and results in a cleaner signal. The accuracy and reliability of this system have been verified in recent studies. ${ }^{11-12}$ Impedance electrodes were placed on the forehead (1), at the left side of the base of the neck (2), the left mid-axillary line at the level of the xiphoid (3), and at the crest of the left hip (4). A phonocardiograph microphone was placed over the left second parasternal region to assist with determination of left ventricular ejection time.

Blood pressure was measured continuously by finger photoplethysmography (Finapres 2300, Ohmeda, Louisville, Colorado). This method provides beat to beat blood pressure values comparable with intra-arterial recordings during postural manoeuvres. ${ }^{13}$

Forearm blood flow was measured by venous occlusion plethysmography using a Silastic strain gauge containing doubled single strand mercury and a rapid cuff inflation system (model E-20) (D E Hokansen, Issaquah, Washington). Continuous monitoring of standard three lead CM5 electrocardiograms was performed.

PROTOCOL

Informed consent was obtained and all participants received instruction in the protocol sequence and the desired position for squatting before performing the study. The study protocol was approved by the University of Wisconsin Medical School Committee on Human Subjects.
Baseline upright measurements were obtained after 3 min of quiet standing with the upper arm maintained on a support stand at estimated right atrial level and the forearm elevated at a slight angle.

Squatting was then performed for a period of $2 \mathrm{~min}$. Body weight was positioned over the heels and a wedge of sponge rubber was placed between the buttocks and heels to prevent possible knee injury due to excessive flexion during squatting. The torso was maintained in a near vertical position, the forearm was held on a supporting stand at the same relative position to the body. Participants were instructed to maintain a normal breathing pattern.

A second trial was performed in each position after $5 \mathrm{~min}$ of recovery from the initial squat to assess repeatability.

ANALYSIS OF HAEMODYNAMIC INDICES

Impedance waveforms were continuously recorded and ensemble averaged over $10 \mathrm{~s}$ intervals. Stroke volume was determined by computer algorithm based on the Kubicek equation ${ }^{14}$ :

$$
\mathrm{SV}=\rho \cdot \frac{\mathrm{L} 2 \cdot \mathrm{T}}{\mathrm{Z}_{0}^{2}}\left(\mathrm{dZ} / \mathrm{dt}_{\max }\right)
$$

where $\mathrm{SV}=$ stroke volume,$\rho=$ patient's blood resistivity (assumed constant at $150 \Omega$ $\mathrm{cm}), \mathrm{L}$ is distance between electrode numbers 2 and $3, T$ is ventricular ejection time, $Z_{0}$ is average baseline impedance, and $\mathrm{dZ} / \mathrm{dt}_{\max }$ is the peak value of the impedance derivative. Cardiac output was then calculated from the product of stroke volume and mean heart rate in beats/min during the $10 \mathrm{~s}$ interval. Cardiac output and stroke volume were automatically converted to cardiac index and stroke index.

We anticipated possible changes in interelectrode distance and baseline $Z_{o}$ during squatting. Small corrections were required for electrode distance (L), but no drift in $Z_{o}$ was observed.

The thoracic fluid volume index was calculated as the reciprocal of the transthoracic impedance. This value provides a dynamic indicator of thoracic fluid volume change. ${ }^{15}$ Cardiac output and forearm blood flow values were time matched with $10 \mathrm{~s}$ averages of beat to beat blood pressure measurement. Mean arterial blood pressure was calculated as diastolic pressure and one third of the pulse pressure value. Total peripheral and forearm vascular resistance were derived from the corresponding ratio between mean arterial pressure and cardiac output or between mean arterial pressure and forearm blood flow.

\section{STATISTICAL ANALYSIS}

Data were analysed for intragroup and intergroup differences by repeated measures analysis with post hoc tests performed using Fisher's protected least squares differences. $\alpha$ was set at $0 \cdot 05$. Comparisons were made at standing rest (immediately before squatting), immediately after squatting (5-10 s), and at 20,60 , and $120 \mathrm{~s}$ of sustained squatting. All values are expressed as mean (one $\mathrm{SE}$ ). 


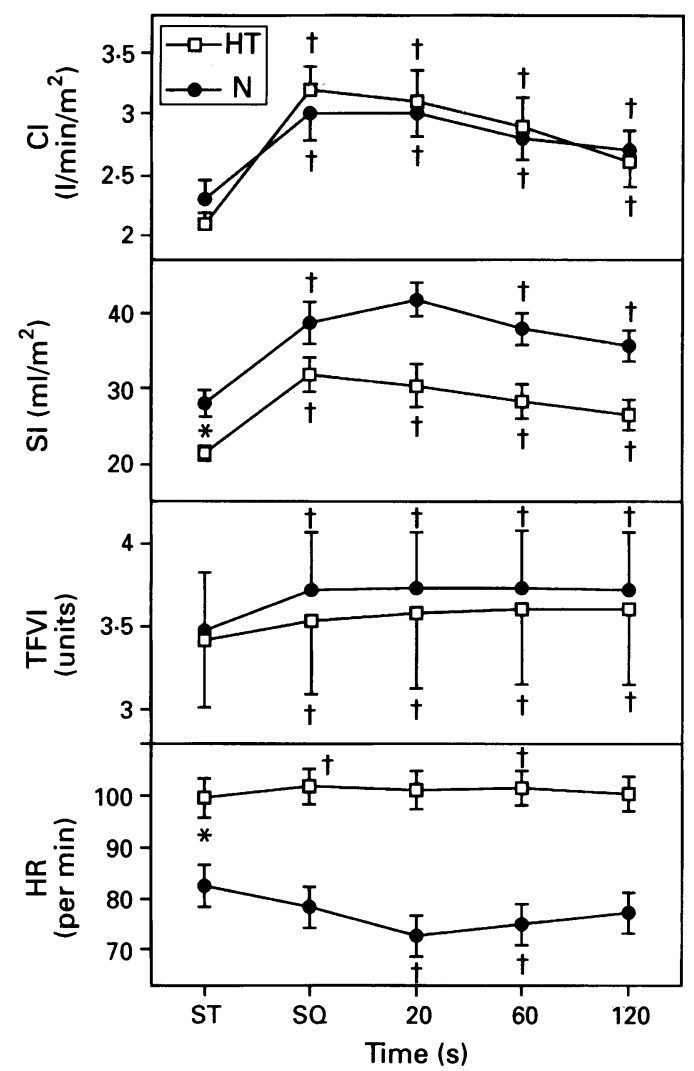

Figure 1 Haemodynamic responses to squatting measured at baseline standing (ST), immediately (5-10 s) after squatting ( $S Q)$, and at 60 , and 120 s of sustained squatting. $N$, normal individuals; $H T$, heart transplant recipients; CI, cardiac index; SI, stroke index; TFVI, thoracic fluid volume index; HR, heart rate.Values are mean (SEM). ${ }^{\star} P=0.05$ between groups. $\dagger P=0.05$ from standing rest.

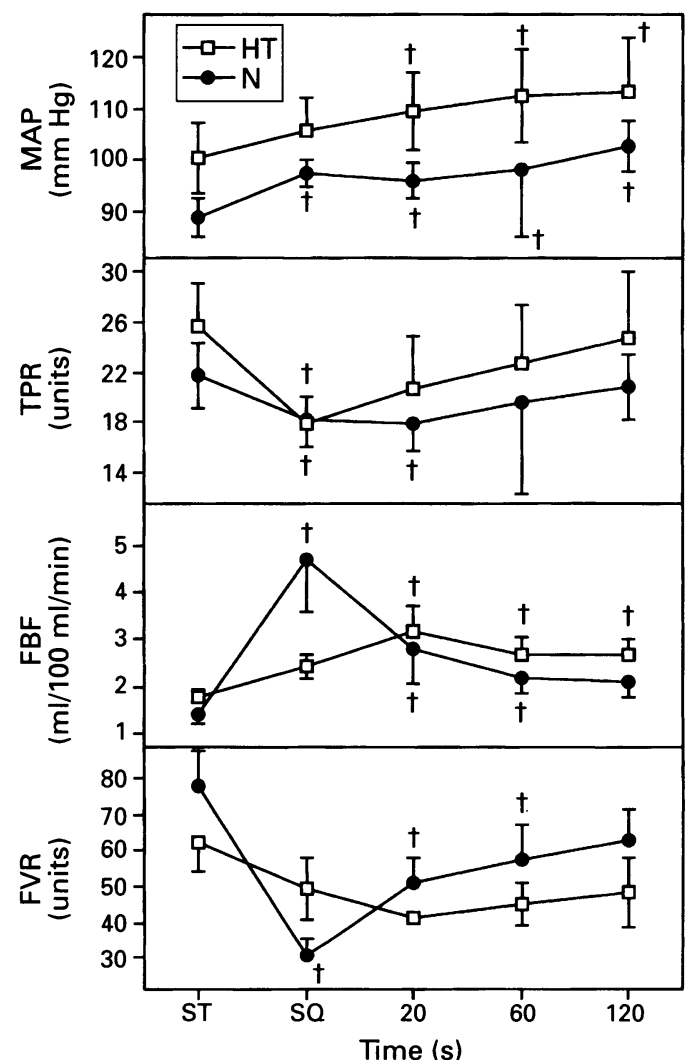

Figure 2 Haemodynamic responses to squatting measured at baseline standing $(S T)$, immediately $(5-10 \mathrm{~s})$ after squatting $(S Q)$, and at 20,60, and 120 s of sustained squatting. $N$, normal individuals; HT, heart transplant recipients; $M A P$, mean arterial pressure; TPR, total peripheral resistance; FBF, forearm blood flow; FVR, forearm vascular resistance. Values are mean (SEM). ${ }^{\star} P=0.05$ between groups. $\dagger P=0.05$ from standing rest.
Results

Figures 1 and 2 show the responses to squatting.

STANDING BASELINE MEASUREMENTS

Values for haemodynamic and derived indices were all within the reported range for normal individuals and heart transplant recipients. ${ }^{5610}$ Heart rate was significantly higher and stroke index significantly lower in heart transplant recipients during standing rest. Otherwise, there were no significant differences between groups before squatting.

\section{RESPONSE TO SQUATTING}

Cardiac index, stroke volume index, and thoracic fluid volume index increased significantly in both groups after the initial $5-10 \mathrm{~s}$ of squatting. The increases in cardiac and thoracic fluid volume indices were similar in normal individuals and heart transplant recipients and the incremental increase in stroke volume index was equal in both groups (normal individuals $10.5 \mathrm{ml} / \mathrm{m}^{2}$ heart transplant recipients $10.3 \mathrm{ml} / \mathrm{m}^{2}$ ). Cardiac and stroke volume indices declined gradually after $20 \mathrm{~s}$ of sustained squatting but remained significantly increased in each group at $120 \mathrm{~s}$. The initial increases in thoracic fluid volume index (normal individuals 0.24 units; heart transplant recipients $0 \cdot 12$ units) remained unchanged in both groups throughout the $120 \mathrm{~s}$ of sustained squatting.

Heart rate in normal individuals declined ( 4 beats/min) at the onset of squatting and was significantly decreased ( 10 beats $/ \mathrm{min}$ ) from $20 \mathrm{~s}$ to $60 \mathrm{~s}$. Heart transplant recipients showed a small but significant increase ( 2 beats/min) in heart rate at the onset of squatting and this increase was maintained for the initial $60 \mathrm{~s}$ of squatting.

Mean arterial pressure increased in both groups at the onset of squatting and exhibited a continuous rise from $20 \mathrm{~s}$ to $120 \mathrm{~s}$ of sustained squatting. The increases in MAP in both groups were significant from $20 \mathrm{~s}$ to $120 \mathrm{~s}$.

Total peripheral resistance decreased significantly in both groups (normal individuals 3.6 units; heart transplant recipients $7 \cdot 7$ units) at the onset of squatting and subsequently returned to baseline values after $20 \mathrm{~s}$ of sustained squatting.

Forearm blood flow increased promptly (3.4 $\mathrm{ml} / 100 \mathrm{ml} / \mathrm{min}$ ) at the onset of squatting in normal individuals. These initial changes declined but remained significantly increased at $60 \mathrm{~s}$ and returned to baseline at $120 \mathrm{~s}$. Heart transplant recipients showed a delayed increase in forearm blood flow which became significant after $20 \mathrm{~s}$ of squatting $(1.4 \mathrm{ml} / 100$ $\mathrm{ml} / \mathrm{min}$ ) and was maintained at this level to $120 \mathrm{~s}$.

Forearm vascular resistance noticeably decreased with initial squatting in normal individuals ( $47 \cdot 8$ units) and remained significantly reduced at $20 \mathrm{~s}$ and $60 \mathrm{~s}$. Heart transplant recipients showed a gradual but non-significant decrease (20.9 units) in forearm vascular resistance during squatting. 
Coefficients of variation for cardiovascular variables during two trials of squatting

\begin{tabular}{lllll}
\hline$C I$ & $S V$ & $H R$ & $F B F$ \\
\hline Normal individuals & $0.055(0.02)$ & $0.068(0.02)$ & $0.041(0.01)$ & $0.30(0.16)$ \\
Heart transplant recipients & $0.052(0.01)$ & $0.052(0.015)$ & $0.01(0.007)$ & $0.17(0.04)$ \\
\hline
\end{tabular}

Values are mean (one SD). CI, cardiac index; SV, stroke volume; HR, heart rate; FBF, forearm blood flow. bly contribute to the sustained rise in arterial blood pressure due to activation of the somatic pressor reflex. ${ }^{22} 23$

The initial decrease in peripheral resistance may in part be caused by regional vasodilatation in the arm and possibly the splanchnic vasculature.$^{24}$ In normal individuals there was a prompt increase in forearm blood flow and a corresponding decrease in forearm vascular resistance, whereas heart transplant recipients exhibited a blunted rise in forearm blood flow and a non-significant decrease in forearm vascular resistance. The impairment in forearm vasodilatation in heart transplant recipients is possibly due to the loss of cardiopulmonary and ventricular baroreflexes which are thought to initiate peripheral vasodilatation during squatting or other forms of increased venous return..$^{24}$ This interpretation is limited, however, by difficulty in determining the relative contributions of cardiopulmonary and sinoaortic baroreflexes during the squatting manoeuvre.

Several other factors may have contributed to the impaired forearm vasodilatation in heart transplant recipients. Early after transplantation recipients may show a blunted maximal hyperaemic muscle blood flow response after ischaemic arterial occlusion. ${ }^{26}$ These abnormalities gradually resolve and probably reflect residual impairment of flow mediated vasodilatation which typically occurs in chronic heart failure. ${ }^{26}{ }^{27} \mathrm{We}$ did not measure maximal forearm blood flow responses to ischaemic occlusion; however, previous studies from our laboratory have shown normal forearm vasodilatation responses 16 months after heart transplantation. ${ }^{28}$ Finally, cyclosporin has been reported to increase sympathetic nervous system activity in heart transplant recipients. ${ }^{29}$ Cyclosporin may possibly have augmented forearm vasoconstrictor tone and diminished reflex peripheral vasodilatation normally associated with squatting.

\section{LIMITATIONS}

A potential limitation of this study is the assumed accuracy of ensemble averaged impedance cardiography for determination of stroke volume during non-steady state haemodynamic responses. We did not verify the measurements of stroke volume and cardiac output by independent methods. Previous studies, however, have reported satisfactory agreement between impedance and thermal or dye dilution methods for determining cardiac output during postural stress, ${ }^{30}$ Valsalva manoeuvre, ${ }^{9}$ and exercise testing. ${ }^{7}$

Medications were not discontinued in heart transplant recipients due to clinical and logistical factors. Three of these patients were treated with antihypertensive agents which could potentially decrease forearm vascular tone. Forearm blood flow and vascular resistance values in the standing position were similar in both groups so it is unlikely that these agents influenced the observed impairment in forearm vasodilatation during squatting. observed during squatting is caused by a rise in peripheral resistance which is attributed to muscular compression of vascular elements in the lower extremities. ${ }^{12}{ }^{19-21}$ Static leg muscle contraction during squatting may also possi- 


\section{Conclusions}

The primary haemodynamic responses to squatting are similar in normal individuals and heart transplant recipients. Each group showed a prompt and sustained increase in cardiac output and arterial blood pressure. The rise in arterial blood pressure was mediated by increased cardiac output as peripheral vascular resistance in both groups decreased initially and subsequently returned to baseline values. These findings are in contrast to existing interpretations which have assumed that peripheral resistance increases during squatting. Normal individuals exhibit a characteristic bradycardia and forearm vasodilatation during squatting which are attributed to combined activation of cardiopulmonary and sinoaortic baroreflexes in response to increased venous return and arterial blood pressure. Bradycardia is absent in heart transplant recipients due to efferent vagal denervation. Instead, a small increase in heart rate was observed which may be caused by stretch mediated chronotropic mechanisms in the transplanted heart- that is, the Bainbridge reflux. Finally, heart transplant recipients show attenuation of the normal decrease in forearm vascular resistance associated with squatting which may be attributed in part to deafferentation of cardiac baroreceptors or localised impairment of flow mediated vasodilatation.

We are grateful to Ms Sharon Vike for expert word processing and Dr Barbara Morgan, Dr Thomas Ebert, and Jill Barney and Dr Barbara Morgan,

1 Sharpley-Shafer EP. Effects of squatting on the normal and failing circulation. $B M 7$ 1956;1:1072-4.

2 O'Donnell TV, Mcllroy MB. The circulatory effects of squatting. Am Heart f 1962;64:347-56.

3 Lance VQ Spodick DH. Physiological responses to prompt and sustained squatting. Br Heart $\mathscr{f} 1977 ; 39$ : $559-62$.

4 VanLieshout J, TenHarkel $\mathrm{AD}$, Wieling $\mathrm{W}$. Physical manoeuvres for combating orthostatic dizziness in autonomic failure. Lancet 1992;339:897-8.

5 Rudas L, Pflugfelder PW, Kostuk WJ. Comparison of haemodynamic responses during dynamic exercise in the upright and supine postures after orthotopic heart transplantation. ₹ Am Coll Cardiol 1990;16:1367-73.

6 Rudas L, Pflugfelder PW, Kostuk WJ. Immediate cardiovascular responses to orthostasis in the early and late months after cardiac transplantation. Int 7 Cardiol 1993; 38:141-50.

7 Muzi M, Ebert TJ, Tristani FE, Jeutter DC, Barney JA, Smith JJ. Determination of cardiac output using ensemble
averaged impedance cardiograms. $₹$ Appl Physiol 1985; averaged im.

8 Ebert TJ, Eckberg DL, Vetroivec GM, Cowley MJ. Impedance cardiograms reliably estimate beat-by-beat changes of left ventricular stroke volume in humans. Cardiovasc Res 1984;18:354-60.

9 Smith SA, Salih MM, Littler WA. Assessment of beat to beat changes in cardiac output during the Valsalva manoeuvre using electrical bioimpedance cardiography. Clin Sci 1987;72:423-38.

10 Starling RC, Binkley PF, Haas GJ, Hatton PS, WoodingScott $M$. Thermodilution measures of right ventricular ejection fraction and volumes in heart transplant recipients: a comparison with radionuclide angiography. $f$ Heart Lung Transplant 1992;11:1140-6.

11 Ebert TJ, Barney JA, Muzi M, Smith JJ. Advanced technology applied to transthoracic impedance measurenology applied to transthoracic impedance measure-
ments improves accuracy and reproducibility of cardiac ments improves accuracy and reproducibility of ca
output determinations. $f$ Clin Monit 1991;7:113-4.

12 de las Alas VR, Tremper KK. Comparison of noninvasive bioimpedance to thermodilution cardiac output. Anesthesiology 1993;79:3A, A471.

13 Parati G, Casadei R, Groppelli A, DiRienzo M, Mancia G. Comparison of finger and intra-arterial blood pressure monitoring at rest and during laboratory testing. Hypertension 1989;13:647-55.

14 Kubicek WG, Kottke FJ, Ramos MU, Patterson RP, Witsoe DA, LaBree JW, et al. The Minnesota impedance cardiograph: theory and applications. Biomed Eng 1974; cardiograph:

15 Ebert TJ, Smith JJ, Barney JA, Merrill DC, Smith GK. The use of thoracic impedance for determining thoracic blood volume changes in man. Aviat Space Environ Med 1986;57:49-53.

16 Snedecor GW, Cochran WG. Statistical methods. 7th ed. Ames, IA: Iowa State University Press, 1980:37.

17 Pathak CL. Autoregulation of chronotropic response of the heart through pacemaker stretch. Cardiology 1973; 58:45-64.

18 Bernardi L, Keller F, Sanders M, Reddy PS, Griffith B, Meno F, et al. Respiratory sinus arrhythmia in the dener-
vated human heart. $₹$ Appl Physiol 1989;67:1447-55.

19 Abrams J. Essentials of cardiac physical diagnosis. Philadelphia: Lea and Febiger, 1987:365.

20 Brotmacher L. Haemodynamic effects of squatting during repose. Br Heart $\mathcal{f}$ 1957;19:559-66.

21 Constant J. Bedside cardiology. 2nd ed. Boston: Little, Brown, 1969:179.

22 Mitchell JH, Wildenthal K. Static (isometric) exercise and the heart. Physiological and clinical considerations. Annu Rev Med 1974;25:369-81.

23 Bezucha GR, Lenser MC, Hanson PG, Nagle FJ. Comparison of haemodynamic responses to static and dynamic exercise. $₹$ Appl Physiol 1982;53:1584-93.

24 Mark AL, Mancia G. Cardiopulmonary baroreflexes in humans. In: Shepherd JT, Abboud FM, eds. The peripheral circulation. The Cardiovascular System, Baltimore: American Physiological Society, 1983:795-813.

25 Mohanty PK, Thames MD, Arrowood JA, Sowers JR, McNamara C, Szentpetery S. Impairment of cardiopulmonary baroreflex after cardiac transplantation in humans. Circulation 1987;75:914-21.

26 Sinoway LI, Minotti JR, Davis D, Pennock JL, Burg JE, Musch TI, et al. Delayed reversal of impaired vasodilaMusch TI, et al. Delayed reversal of impaired vasodilation in congestive heart failure after
tion. Am $\mathcal{F}$ Cardiol 1988;61:1076-9.

27 Wilson JR, Martin JL, Schwartz D, Ferraro N. Exercise intolerance in patients with chronic heart failure: role of impaired nutritive blood flow to skeletal muscle. Circulation 1984;69:1079-87.

28 Morgan BJ, DeBoer LVW, Pease MO, Scherrer U, Vissing $\mathrm{SF}$, Hanson P. Forearm vascular resistance increases during static exercise in heart transplant recipients. $\mathcal{F}$ Appl Physiol 1991;71:2224-30.

29 Scherrer U, Vissing SF, Morgan BF, Rollins JA, Tindall RSA, Ring S, et al. Cyclosporine induced sympathetic activation and hypertension after heart transplantation. activation and hypertension after

30 Smith J, Bush JE, Wieldmeir VT, Tristani FE. Application of impedance cardiography to study of postural stress. F Appl Physiol 1970;29:133-7. 